\title{
La dimensión educativa como incipiente valor añadido en los portales periodísticos
}

The educative dimension as a new value added in the journalistic portals

\author{
Francisco Javier Martínez Méndez (1) y Rosana López Carreño (2) \\ Departamento de Información y Documentación, Facultad de Comunicación y Documentación, \\ Universidad de Murcia, España, (1) javima@um.es (2) rosanalc@um.es
}

\begin{abstract}
Resumen
El trabajo analiza la incipiente faceta educativa de los portales periodísticos. Estas sedes se han afianzado como uno de los sitios más visitados de la web y las diferencias en su oferta vienen cada días más marcadas por el grado de desarrollo de sus servicios de valor añadido. Estos servicios comienzan a incorporar las tecnologías de software social, tan corrientes en la Web 2.0. Una de sus aplicaciones, aún en un nivel de desarrollo pequeño, es la promoción de actividades educativas y culturales por medio de este nuevo tipo de servicios. Si bien podemos encontrar interesantes ejemplos de alfabetización lingüística y cultural a nivel internacional, la implantación en España es aún escasa. No obstante, la vinculación entre los medios de comunicación y el sector editorial unida a la fidelidad de sus usuarios parece indicar que se trata de un campo de inminente e imparable evolución.
\end{abstract}

Palabras clave: Portales periodísticos. Educación. World Wide Web. Software Social. Enseñanza de Idiomas.

\section{Introducción}

La presencia de los medios de comunicación en Internet se ha consolidado a través del éxito de los distintos portales periodísticos. Si bien para algunos esta hecho pueda parecer novedoso, hace ya más de diez años que se implantaron las primeras sedes web (entonces ediciones digitales de las principales cabeceras periodísticas). Aunque el grado de desarrollo es diverso, hace ya tiempo que los principales medios de comunicación (incluyendo radio y televisión) han afianzado sus sistemas de información en la web constituyendo verdaderos portales. Casi todos ellos coinciden en la oferta de productos informativo-documentales y en la disponibilidad de servicios básicos de acceso a la información y a la documentación. En los últimos tiempos los principales portales apuestan por la diversificación de su oferta, tanto en el campo de lo social como de la extensión cultural y, de forma quizá

\begin{abstract}
This article analyses the recent educational aspects which are present in journalistic portals. These sites are the most visited in the web and the differences in their offer are marked by the development of value added services. These services begin to add social software technology, typical of the Web 2.0. One of their applications, still in a low level of development, is the promotion of educational and cultural activities. Although we can find interesting examples in the field of linguistic instruction and cultural education at an international level, their level of development in Spain is still very poor. However, the latent affinity between mass media and the publishing sector-together with the fidelity of their users-augurs very interesting and promising perspectives to this field of research and development.
\end{abstract}

Keywords: Journalistic portals. Education. World Wide Web. Social Software. Languages Instruction.

algo incipiente, en el campo de lo educativo. Es decir, nos encontramos en un momento de desarrollo de los portales periodísticos donde las diferencias entre un medio y otro no se asientan únicamente en la oferta informativo-documental, sino también en las funciones adicionales del portal.

La mayor plataforma de comunicación mundial, la web, potencia la evolución de la Sociedad de la Información hacia la Sociedad del Conocimiento y éste puede ser un ejemplo de una tendencia cada vez mayor de implantación y desarrollo de todo tipo de portales dotados de un amplio abanico de herramientas y servicios de carácter eminentemente educativo. Hace más de treinta años que quedó claro el importante papel que pueden desarrollar los medios de comunicación como apoyo auxiliador en los niveles de educación básica y actualmente (Martínez-Salanova, 2007): 
[la] tecnología convierte los medios en mucho más que meros auxiliares del profesor. Los audiovisuales, otros medios relacionados con ellos, los medios de comunicación en general y los que se basan en tecnología informática, son mucho más que simples auxiliares. Los medios de esta clase constituyen frecuentemente la vanguardia del progreso educativo y cuando se usan con propiedad afectan la estructura de planes y programas y lo que es más, llegan a modificar la estructura íntima del proceso de aprendizaje. Estos medios son esenciales para lograr una comunicación efectiva en la enseñanza a grandes grupos y son el único medio de comunicación didáctica en muchos programas para enseñanza individual o estudio independiente.

Es una realidad evidente que "el contexto naciente de una cultura digital repercute sobre la acción educativa y el sistema educativo" (Pérez Tapia, 2006), pero también lo hace en otros ámbitos educativos no reglados, como es el caso de la web y, dentro de ella, también lo encontramos en el seno de los portales periodísticos con interés social por el fomento educativo-cultural de sus contenidos. Muchos autores han llegado a afirmar que la educación a través de los medios de comunicación podría llegar a alcanzar el nivel de un nuevo paradigma educativo, así Morduchowicz (2001) afirmaba que

hay quienes suelen hablar de una primera alfabetización, protagonizada por el libro y la cultura impresa, y una segunda que nos abre a las múltiples escrituras que hoy conforman el mundo audiovisual e informático. Estos cambios no significan la sustitución de un modo de leer por otro, sino la compleja articulación de uno y de otro, de la lectura de textos y de hipertextos.

Bajo esta perspectiva es posible encontrar algunas líneas de desarrollo complementarias de los portales periodísticos ligeramente ajenas de la mera (y principal) función informativa.

\section{La así llamada "web 2.0"}

Son muchos los autores que afirman que estamos inmersos de pleno en la denominada "web 2.0". Es bien sabido que este término fue acuñado en medio de una "tormenta de ideas" dirigida por Dale Dougherty (vicepresidente de la consultora O'Reilly Media) para desarrollar una sensación basada en un cierto "renacimiento" de la web. Esta evolución para algunos (entre los que nos posicionamos) no es tan profunda, sino que es una continuación lógica sin mayor ruptura de la web original implementada a principios de los años noventa por Tim Berners-Lee, progreso que se ha visto ayudado por el espectacular crecimiento de los anchos de banda de las líneas de comunicaciones (lo que permite, entre otras muchas posibilidades, habilitar streamings de vídeo de hasta 100 megabtes, base del éxito de youtube.com, uno de los servicios estrella de esta "web 2.0") y el no menos trascendente abaratamiento de los dispositivos de almacenamiento maviso que hacen posible la puesta en funcionamiento de plataformas de correo electrónico con capacidades de memoria hasta hace pocos años inimaginables (es el caso de los servicios de correo electrónico gratuitos y con varios gigabytes de gmail o hotmail).

No obstante, nuestra opinión es realmente minoritaria y el uso del término es algo común y frecuente, no se sabe todavía si más por razones de marketing que por otra causa. Algunos piensan que puede ser una reedición de la llamada "burbuja tecnológica" de finales de los años noventa, aunque otros como Serrano Cobos (2006) dicen que es precisamente la "web post burbuja 2000", aquella en la que los servicios han adquirido fortaleza gracias a su elevado carácter colaborativo y participativo. Independientemente de esta discusión, es un hecho innegable que tanto en la literatura científica como en la técnica su uso está plenamente aceptado y no parece que se vaya a quedar ahí porque continúa extendiéndose a otros entornos, alguno tan cercano a nuestro campo como el de "biblioteca 2.0" que (entre otras cosas), va a fomentar la participación del usuario no sólo en el uso de la biblioteca sino también en su gestión, y socializa la arquitectura y posterior recuperación de información, permitiendo descentralizar la gestión de contenidos (Serrano Cobos, 2006).

La figura 1 representa un pequeño mapa mental ("webmeme" es el término acuñado dentro de la "web 2.0" para estos mapas) desarrollado a partir de los servicios y las tecnologías empleadas en este nuevo contexto. Un mapa visual mucho más desarrollado y actualizado ha sido publicado por la Fundación Orange (Internality, 2007). Dejando a un lado la mera discusión sobre la conveniencia del término, parece evidente que han cambiado las tendencias en la web, pasando la misma de ser un entorno donde los gestores de contenidos ubicaban información para su consulta y una posterior ( $\mathrm{y}$ residual en términos porcentuales) redifusión de esta información vía correo electrónico, a un entorno mucho más dinámico donde todos los usuarios potencialmente pueden convertirse en gestores de contenidos sin muchos problemas tecnológicos ni económicos, gracias especialmente a los blogs, wikis, la sindicación, el etiquetado (en definitiva, a todo el conjunto de aplicaciones que se agrupan bajo la idea de software social) convirtiéndose los usuarios de la web en promotores activos de la difusión de la información y del conocimiento en ella almacenada. 


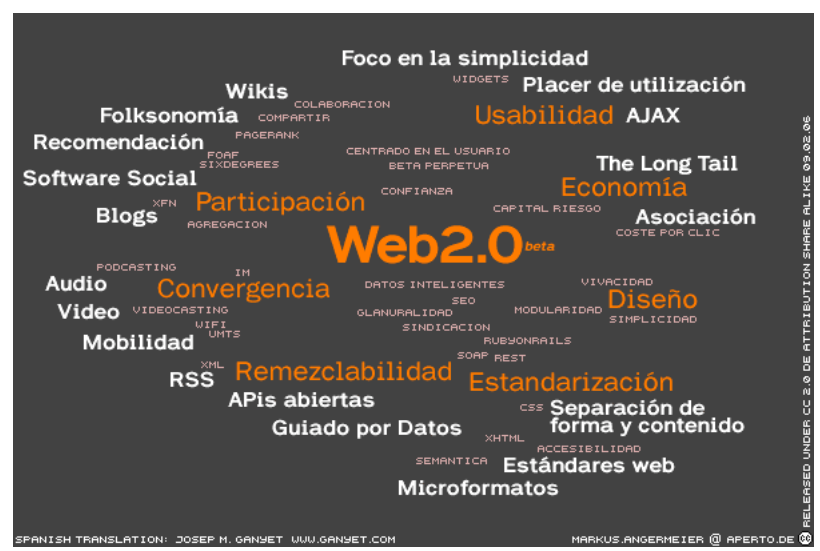

Figura 1. Webmeme elaborado por Markus Angermeier que resume la idea de Web 2.0, con algunos ejemplos de servicios. Fuente: Wikipedia (<http://es.wikipedia.org/wiki/Web_2.0>).

El que este nuevo contexto sea mucho más colaborativo y asociativo que el anterior no es sólo un efecto de las nuevas aplicaciones tecnológicas; es también una nueva forma de saber ser y saber estar en la web, es decir, es una competencia que se ha adquirido y que se viene aplicando de forma masiva. La propia Unión Europea a la hora de definir las competencias claves para el aprendizaje permanente destaca entre ellas "la capacidad de buscar, obtener y tratar información, así como de utilizarla de manera crítica y sistemática, evaluando su pertinencia y diferenciando entre información real y virtual, pero reconociendo al mismo tiempo los vínculos. Las personas deben ser capaces de utilizar herramientas para producir, presentar y comprender información compleja y tener la habilidad necesaria para acceder a servicios basados en Internet, buscarlos y utilizarlos, pero también deben saber cómo utilizar las TSI en apoyo del pensamiento crítico, la creatividad y la innovación" (UE, 2006). Nos encontramos conque "hay un sentido lírico en la alfabetización tecnológica y en la propia metodología cuando las ideas se transmutan en actividades y éstas en un proyecto colectivo que las transciende para ser tomadas colectivamente por la ciudadanía libremente organizada" (Junta de Extremadura, 2005), este entorno es además mucho más democrático.

\section{Las tendencias educativas y sociales en la web}

Hasta ahora las apuestas educativas en la web consistían en el desarrollo de grandes portales educativos, la mayoría de ellos auspiciados por las administraciones públicas en el entorno de sus competencias. López Carreño y Bunes Portillo (2007) desarrollan con suficientes ejemplos el esquema inicial aportado por Area Moreira (2003):

\begin{tabular}{|l|l|}
\hline \multicolumn{2}{|l|}{ Portal Educativo } \\
\hline Informativos & $\begin{array}{l}\text { Institucionales o de grupos } \\
\text { educativos. }\end{array}$ \\
\cline { 2 - 2 } & De recursos educativos. \\
\hline Formativos & $\begin{array}{l}\text { Entornos de formación e intranets } \\
\text { educativas. }\end{array}$ \\
\cline { 2 - 2 } & Repositorios de material didáctico. \\
\hline
\end{tabular}

Tabla I. Tipos de portales educativos según Area Moreira (2003).

Independientemente del grado de desarrollo y propósito de estos sitios web, en todos ellos se detecta una limitación: la ausencia de participación del usuario. Hasta ahora el mismo se ha limitado a adquirir un conocimiento dentro de un curso a distancia o por medio de la consulta de alguna documentación en línea dentro un portal institucional, todo ello dentro de un itinerario más o menos fijado de antemano por los gestores de los portales y los diseñadores de los cursos, lo más independiente que venía a realizar podría ser la descarga de alguna aplicación o documentación dentro de un repositorio de materiales didácticos. La interactividad y comunicación con compañeros se limitaba a la participación en foros y debates, generalmente dentro del entorno reducido de un curso, cuya trascendencia exterior era escasa o, más bien, nula.

El software social, entendido como respaldo, amplía y genera valor del comportamiento social de los seres humanos e incrementa el valor de todos estos recursos. Es, en definitiva, un servicio de valor añadido que complementa la ingente tarea desarrollada en el seno de los portales educativos, al igual que hace en otros entornos. Las aplicaciones más destacadas son:

- Foros: Servicio ya disponible en la web tradicional pero de indudable valor cooperativo y social.

- Grupos: Evolución de los tradicionales servicios de 'news' especializados en una temática determinada (yahoo.groups y google.groups acogen la mayoría de estos servicios, desarrollados muchos de ellos incluso antes de la creación de la web).

- Wikis: Es un sitio web colaborativo que puede ser editado por varios usuarios y que permite la edición compartida de artículos. El mejor ejemplo de esta tecnología es Wikipedia, biblioteca en línea gratuita creada por Jimmy Wales (1) y desarrollada de forma colaborativa por cientos de miles de editores en todo el 
mundo. También podemos encontrar archivos wikis destinados a la formación en el uso de una tecnología o bien para la exposición de los materiales didácticos de una asignatura (como si de un tutorial se tratara), desarrollados generalmente por medio de la aplicación TiddlyWiki.

- Blogs: También conocidos como "bitácoras" o diarios personales en línea. Estos servicios han revolucionado la edición de contenidos en la web y han modificado sustancialmente la difusión de los mismos. Muchos de estos blogs se están convirtiendo en sitios de referencia para el aprendizaje (sirva como ejemplo el proyecto economia.com desarrollado para la enseñanza de la Economía en Enseñanza Secundaria) y para la actualización de contenidos (uno de los más visitados por los interesados en recuperación de información en la web es el blog de Matt Cutts, Ingeniero Jefe de Google y responsable de las búsquedas en el motor). La flexibilidad de edición de estos diarios personales no se limita al autor, sino que todos los lectores pueden interactuar con ellos por la vía de los comentarios. Se llegan a formar verdaderas comunidades de blogs interesados en una temática y son los editores de un blog los que comentan artículos y noticias publicados en otros; propagándose toda esta información de forma absolutamente interactiva y personalizada gracias a la sindicación de contenidos, que ha demostrado en este campo su potencialidad.

- Folksonomías: según Wikipedia, este término "es un neologismo que da nombre a la categorización colaborativa por medio de etiquetas simples en un espacio de nombres llano, sin jerarquías ni relaciones de parentesco predeterminadas. Se trata de una práctica que se produce en entornos de software social cuyos mejores exponentes son los sitios compartidos como del.icio.us (enlaces favoritos), Flickr (fotos), Tagzania (lugares), flof (lugares) o 43 Things (deseos)". Estos sistemas de etiquetado no pueden pasar desapercibidos en nuestro campo porque precisamente son los sistemas que están permitiendo incorporar a la web tradicional unas (eso sí, incipientes aún) dosis de contenido semántico sobre los recursos digitales publicados en la web.

- Mapas: los mapas de vistas satelitales accesibles desde la web están contribuyendo indiscutiblemente a que todos conozcamos un determinado paraje, una ciudad e incluso una determinada ruta con sus lugares más destacados.
- Vídeos en la red: la gran profusión de materiales audiovisuales publicados en la red está modificando sustancialmente la enseñanza en los campos de la Publicidad y el Marketing, no sólo como medio para la difusión de las actividades de ese campo sino también como medio de apoyo para la docencia (actualmente un profesor de Publicidad no necesita copiar en vídeo un anuncio para comentarlo en clase), lo puede incrustar en su blog y comentarlo con todos sus alumnos).

\section{La presencia de servicios con dimensión educativa en los portales periodísticos}

Mestres (2007) afirma que "nadie discute la importancia de los medios de comunicación en la sociedad actual" caracterizada como mediática en la que nuestro estilo de vida convive con los mensajes transmitidos por los medios de comunicación. Estos medios (Mestres, 2007):

constituyen un aparato de socialización muy importante: influyen en nuestras ideas, hábitos y costumbres. Algunos expertos incluso llegan a afirmar que la cantidad de información comunicada por la prensa, las revistas, las películas, la televisión y la radio, excede en gran medida al volumen de información que llega a través de la enseñanza y los contenidos que se transmiten en la escuela.

Así, los medios de comunicación se convierten en una herramienta educativa muy poderosa, con las ventajas y desventajas que esto supone, aunque no sea el educar su finalidad única ni última.

Dentro de este proceso de socialización, adopta, como no podía ser de otro modo, un papel muy destacado el software social, que contribuye a desarrollar servicios de valor añadido, los elementos que - según avanzaba ya López Carreño en su tesis doctoral (2003) - sirven para "distinguir fundamentalmente a los portales periodísticos unos de otros por su oferta". Es precisamente en la aplicación de este software social donde encontramos algunos ejemplos que nos permiten ilustrar la dimensión educativa y social de los portales periodísticos, ya que los mismos no escapan a su aplicación, sino todo lo contrario, porque han sido de los primeros en introducirlos.

No podemos olvidar que los portales periodísticos son de los que mayor número de visitas reciben todos los días junto con los motores de búsqueda (los datos de Nielsen Netratings de febrero 2007 sitúan a los portales del Grupo Prisa entre los diez compañías más visitadas de España, y no podemos olvidar la considerable capacidad informativa de los portales Terra 
Networks, MSN y Yahoo!, situados también en esa lista en lugares aún más altos), es decir, se trata de sedes web totalmente permeables a lo que ocurre en la web y por ello estos portales periodísticos no son ajenos a esta socialización, sino todo lo contrario, a pesar de que contribuyen a su desarrollo: Así, El Mundo introdujo el uso de blogs como sustitutos de las tradicionales columnas de opinión y también hace tiempo que dispone de un traductor en línea; El País aloja diarios personales de los usuarios en la sección "Yo periodista" y permite la redifusión y compartición de sus contenidos por medio de los servicios trackbacks (2), delicious (3), digg (4), technorati (5), Mi yahoo (6) y meneame (7)) de sus blogs por medio de aplicaciones contenidos y casi todos los portales importantes han incorporado la sindicación de contenidos.

Si bien la faceta social está más o menos definida en el fomento de la participación y en la sindicación, no está aún tan desarrollada la faceta educativa, que se manifiesta fundamentalmente en algunas aplicaciones alfabetizadoras. A modo de síntesis podemos afirmar que esta labor educadora se centra en dos tipos de alfabetizaciones claramente diferenciadas: la lingüística y la cultural. En la primera se desarrollan herramientas y servicios de enseñanzaaprendizaje de idiomas de carácter autodidáctico, basados en la reutilización de los contenidos generados por medio de comunicación para su uso en el aprendizaje de un idioma determinado. En el caso de la alfabetización cultural, los portales periodísticos optimizan sus contenidos multimedia para el fomento de la cultura a través de la elaboración de reportajes sobre monumentos, parajes, eventos, etc. En esta línea encontramos la frase de Marc Augé que recoge Tiscar Lara (2007) en su blog: "los medios de comunicación deben ser objeto de educación, no sólo un canal de información". A continuación se muestran ejemplos de estos dos tipos de alfabetizaciones impulsadas desde los portales periodísticos.

\subsection{BBC Learning English}

Desde el punto de vista de la alfabetización lingüística, y más concretamente en el campo del aprendizaje de idiomas, sobresale sin lugar a dudas, este portal vertical del grupo de comunicación británico British Broadcasting Corporation que nació en 1996 y que se constituyéndose como el referente de este tipo de iniciativas educativas impulsadas por medios de comunicación en la red y fuente de información y contenidos dónde muchas instituciones educativas de enseñanza de idiomas se nutren para reforzar sus programas educativos reglados.
Desde este sitio web (sito es <http://www.bbc.co .uk/worldservice/learningenglish/>), no sólo se puede aprender inglés a través de los contenidos reales del grupo de comunicación (prensa, radio y $T V$ ), sino que también ofrece la posibilidad del aprendizaje de otros idiomas, " $B B C$ Languages" como son el español, francés alemán, italiano, portugués, griego y chino mandarín. Aunque, evidentemente, el idioma con mayor tratamiento es el inglés, ya que es la lengua habitual del grupo de comunicación.

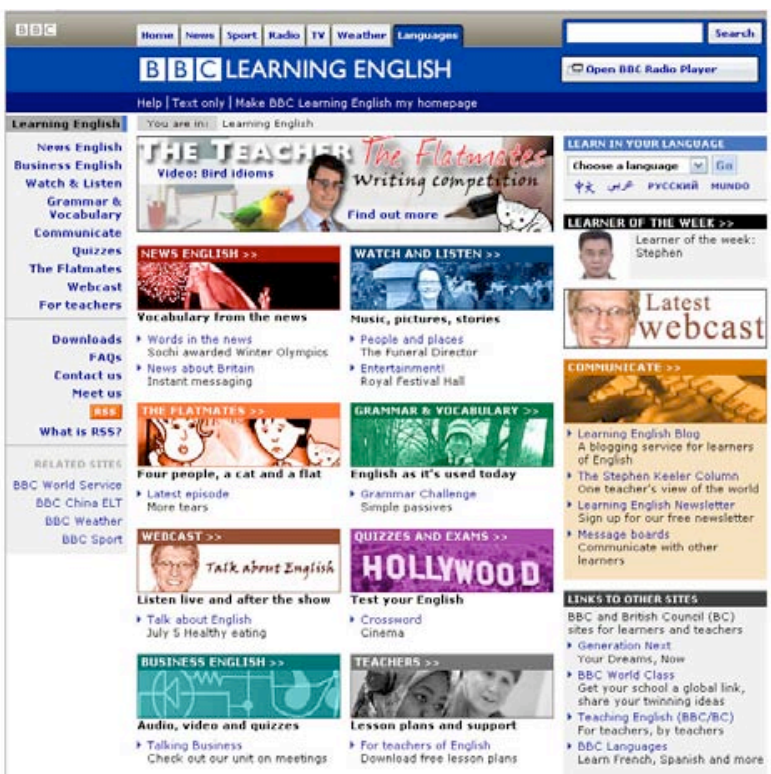

Figura 2. Sección del portal BBC Learning English.

Aunque sus autores denominen "servicio" a esta oferta educativa, su desarrollo es tan amplio que se trata verdaderamente de un portal con una amplia gama de productos y servicios que se encuadran en los siguientes apartados:

- News English: Esta sección ayuda a mejorar y conocer vocabulario a través de definiciones y explicaciones de términos o frases del inglés, destacados en textos de noticias publicadas. Cuenta con un amplio archivo de documentos desde 2001 hasta la actualidad, publicando tres nuevos contenidos por semana. Además ofrece un apartado, "News about Britian" que profundiza en las costumbres y cultura del Reino Unido. Las destrezas a desarrollar están orientadas a la compresión de textos ("readings") -ya que puedes leer el texto y descargar material complementario de ejercicios sobre el mismo- y comprensión oral ("listenings") - permiten la descarga de ficheros de audio. Estas herramientas se consideran vitales en el aprendizaje de un idioma. 
- Business English: Se ofrece material específico (casos prácticos e información) para el uso del inglés en el ámbito laboral por medio de audiovisuales y juegos.

- Watch \& Listen: En este apartado se intenta mejorar la compresión oral del ingles a través de ejercicios test y prácticas completados con ficheros de audio y vídeo. Además, se ofrece la posibilidad de descargar la trascripción de conversaciones en documentos PDF. Ofrece contenidos sobre música, ocio, cultura y la vida cotidiana en Londres, además de facilitar mini lecciones con audio y ejercicios sobre la pronunciación del inglés.

- Grammar \& Vocabulary: En esta sección se pueden encontrar muchas respuestas sobre dudas en el uso del inglés. Se oferta tanto gramática básica como vocabulario general y específico en temas (deportes, ciencias, etc.).

- Comunicate: Es el apartado más participativo ya que el usuario puede chatear con otros usuarios, tomar parte en foros, bitácoras y tablones de anuncios.

- Quizzes: Desde esta sección se ofrece un conjunto de juegos basados en el lenguaje (por ejemplo, crucigramas o encontrar una palabra) y temas de interés, así cómo ejercicios basados en exámenes de idiomas.

- The Flatmates: En este apartado se pueden escuchar episodios basados en diálogos de la vida real, dónde se puede descargar el audio y leer el guión del mismo.

- Webcast: En este apartado se pueden escuchar programas de radio donde se tratan diferentes temas y donde también se ofrece la posibilidad de descargar el audio y el texto.

- For teachers: Esta sección está destinada al profesorado de enseñanza del inglés, donde puede obtener material que puede utilizar en sus clases e intercambiar ideas sobre la docencia con otros docentes.

Además en $B B C$ Learning English se ofrece una serie de servicios que ayudan al usuario a acceder a la información, como sindicación de contenidos (vía RSS) y descarga de ficheros de audio, vídeo y documentos en formato PDF.

\subsection{Radio Praga}

A través de su versión en español, esta emisora de la República Checa ofrece unas lecciones básicas de gramática y vocabulario checo a través de su apartado "Hable Checo con Radio Praga" (sito en <http://www.radio.cz/es/actual/ hable>) donde se puede leer el texto en español de temas sobre la vida que se actualizan semanalmente, ver la comparación de términos en checo, así como escuchar el audio y descargar la trascripción del mismo en formato PDF. Al igual que en el caso anterior también oferta sindicación de contenidos vía RSS. Aunque constituye una iniciativa más modesta que la del portal de la BBC, constituye una muestra de la intencionalidad por parte de los portales periodísticos de reutilización de sus contenidos en la enseñanza-aprendizaje de idiomas.

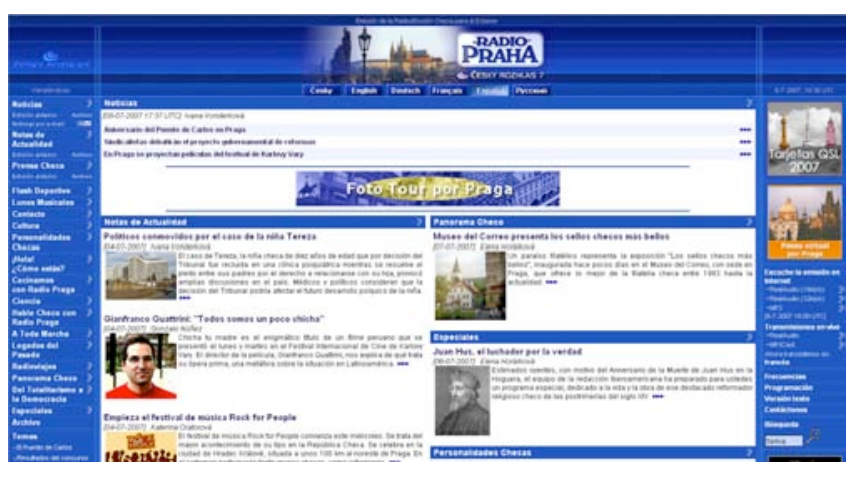

Figura 3. Sección del portal educativo de Radio Praha.

\subsection{Discovery Channel}

Esta cadena de televisión por cable especializada en reportajes de gran calidad ofrece desde su portal una gran variedad de material audiovisual en distintas materias que ayudan al usuario a profundizar en temas como zoología, viajes, salud, ciencias, etc. por medio de sus canales o portales verticales especializados (podemos verlo en <http://www.discovery.com/>).

Todos los portales verticales dependientes de "Discovery.com" presentan un diseño genérico y ofertan los mismos servicios: foros, RSS, vídeos, juegos, galería de fotos, etc. Además ofrece una versión en castellano de los contenidos más relevantes del portal periodístico. Este sitio web constituye una versión online de los tradicionales documentales televisivos que optimizan la alfabetización cultural, a nivel general, entre el gran público de la red; y se convierte en una valiosa fuente de recursos audiovisuales para las disciplinas de Ciencias e Historia de la enseñanza reglada. 


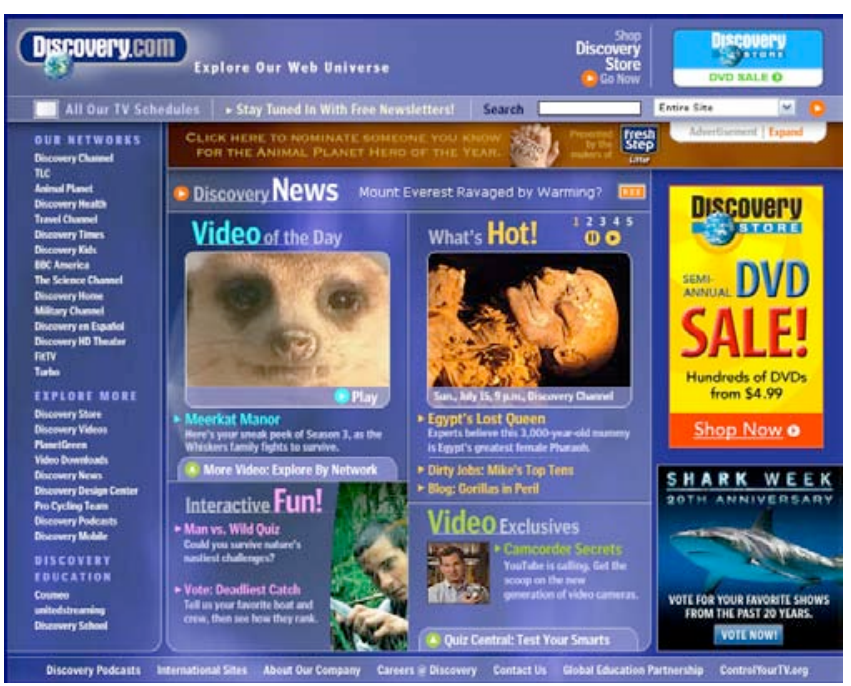

Figura 4. Sección del portal Discovery Channel.

\subsection{EP3}

El portal vertical cultural del diario El País es un claro ejemplo de la apuesta por parte de los medios de comunicación españoles en el fomento de la cultura en cualquiera de sus vertientes: música, cine, teatro, exposiciones, etc.

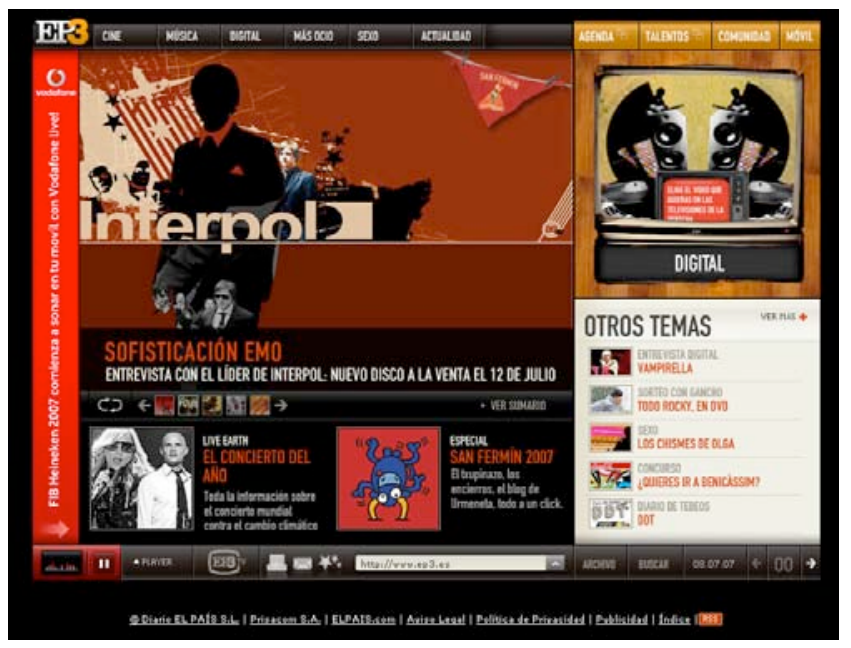

Figura 5. Sección del portal EP3.

Este sitio (accesible en <http://www.ep3.es/>) presenta una incipiente introducción en el desarrollo de contenidos y servicios de valor añadido que ofertan, entre los que destacan blogs, TV, radio, RSS, vídeos, concursos y agenda de eventos.

\section{Conclusiones}

A modo de reflexión general se pueden adelantar las siguientes conclusiones:
1. Los portales periodísticos son sitios web permeables a la actual corriente social que impera en la web, y se surten de sus elementos y aplicaciones para el desarrollo de nuevos servicios de valor añadido. Algunos de estos elementos les sirven para fomentar facetas educadoras y, evidentemente, sociales.

2. Los servicios derivados de la tecnología de software social se encuentran plenamente afianzados en los principales portales periodísticos.

3. Los portales periodísticos españoles aún no presentan el desarrollo alcanzado por los portales periodísticos británicos en materia educativa y cultural, aunque hayan comenzado a integrar herramientas y servicios auxiliares que promuevan esa función educativa-cultural que pretenden desarrollar.

4. Resulta también lógico que el mejor ejemplo que podemos encontrar de alfabetización lingüística esté ubicado en el portal de la BBC, ya que este grupo editorial es uno de los más prestigiosos en la enseñanza de idiomas a distancia. Este portal vertical para el aprendizaje de idiomas, es actualmente una fuente vital de contenidos utilizados en la enseñanza de idiomas en los sistemas educativos de muchos países. Sus contenidos abarcan las cuatro destrezas fundamentales para el aprendizaje de un idioma: compresión oral, compresión escrita, expresión oral y expresión escrita.

5. Llama poderosamente la atención (en sentido negativo) que el nivel de desarrollo español en este campo sea escaso cuando algunos portales están también vinculados a prestigiosas editoriales (El País a Santillana o Antena 3 a Planeta DeAgostini, éste último competidor directo de $\mathrm{BBC}$ en esa área de negocio).

6. Si bien el nivel de desarrollo alcanzado hasta ahora es bajo, no se puede dejar de lado la importancia de nuestro idioma porque (según wikipedia) el español es la segunda lengua en número de hablantes nativos en el mundo tras el chino mandarín, con más de 450 millones de personas que lo utilizan como primera o segunda lengua, aunque hay cifras que alcanzan los 500 millones. Es el tercer idioma más hablado contando a los que lo hablan como primera o segunda lengua, por detrás del chino mandarín y del inglés. Asimismo es el segundo idioma más estudiado en el mundo tras el inglés, con al menos 40 millones de estudiantes distribuidos en 90 países. Estamos convencidos de que en breve, algunos portales periodísticos espa- 
ñoles seguirán la senda iniciada por la BBC. Tampoco se puede dejar de lado la potencialidad de este tipo de aplicaciones para el aprendizaje del resto de lenguas cooficiales del territorio español en portales periodísticos regionales.

7. Es lógico que los esfuerzos en el uso de la información estén enfocados a la alfabetización lingüística fundamentalmente, dado que este hecho fomenta los vínculos de fidelidad de usuarios potenciales de otras lenguas diferentes del inglés; lo que abarca una clara intencionalidad de afianzar la fidelidad de usuarios potenciales al portal periodístico.

8. A pesar de la no muy alentadora situación actual, estamos convencidos de que a pesar de encontrarnos todavía en un estadio inicial, los servicios de valor añadido orientados a promover su faceta educativa serán uno de los elementos distintivos de la oferta de los portales periodísticos en un futuro inmediato. Basamos nuestra tesis en la audiencia e inserción de estos medios, en la competencia por afianzar y personalizar esta audiencia, en la vinculación de algunos de ellos a grandes grupos editoriales y a la potencialidad de nuestro idioma y nuestra cultura en el mundo, que encontrarán en estos medios un terreno abonado para su propagación.

\section{Notas}

(1) Wikipedia es un proyecto para escribir comunitariamente enciclopedias libres en todos los idiomas. Fue fundada por Jimmy Wales y Larry Sanger basándose en el concepto "wiki" que permite cargar colectivamente documentos web, sin que la revisión del contenido sea necesaria antes de su aceptación para ser publicado en la red. La versión en inglés comenzó el 15 de enero de 2001. Su versión es español está ubicada en <http://es.wikipedia.org/wiki/Portada>.

(2) Un trackback es un enlace inverso que permite conocer qué enlaces apuntan hacia un determinado post; de ese modo avisa a otro blog que se está citando uno de sus posts. Si un blog admite trackbacks quiere decir que es capaz de recibir un aviso de otro blog, de forma que dos de los artículos de ambos quedan relacionados entre sí, normalmente porque el segundo hace referencia al primero. Es un claro servicio de socialización.

(3) Del.icio.us es un servicio de gestión de marcadores sociales en web. Permite agregar los marcadores que clásicamente se guardaban en los navegadores y categorizarlos con un sistema de etiquetado.

(4) Digg es un sitio web con énfasis en noticias sobre ciencia y tecnología. Combina marcadores, blogs y sindicación con una organización sin jerarquías, con control editorial democrático.

\section{Referencias}

Area Moreira, Manuel (2003). De los webs educativos al material didáctico web. // Comunicación y Pedagogía, $\mathrm{n}^{\circ}$ 188, 2003, pgs. 32-38. [En línea]. <http:// webpages.ull.es/users/manarea/Documentos/sitiosweb.pdf> [18 junio 2007]

(Junta de Extremadura, 2005) Manual de Alfabetización Tecnológica. [En línea]. Ediciones Simbióticas, 2002. <http://www.edicionessimbioticas.info/article.php3?id_art icle $=401>$ [ 8 julio 2007]

Lara, Tíscar. (2007). Alfabetización digital: una asignatura pendiente. [En línea]. En tiscar.com, 2004. $<$ http://tiscar.com/2007/06/25/alfabetizacion-digital-unaasignatura-pendiente/> [10 julio 2007]

López Carreño, Rosana (2003). Análisis de los portales periodísticos españoles. Taxonomía de sus elementos componentes. [Tesis Doctoral]. [En línea]. Alicante: Biblioteca Miguel de Cervantes, 2003. <http://www. cervantesvirtual.com/FichaObra.html?Ref=10572> [12 julio 2007]

López Carreño, Rosana et al. (2007). Manual de Información y Documentación Educativa. Murcia: Consejería de Educación y Universidades, 2007. [En prensa].

Internality (2007). Mapa visual de la Web 2.0 [En línea] Internality: Madrid, 2007. <http://www.internality.com/ web20/> [2 julio 2007]

Martínez-Salanova Sánchez, E. (2007). Aprender a aprender con los medios de comunicación. [En línea] Huelva: Universidad, 1995. <http://www.uhu.es/cine.educacion/ didactica/0061aprenderconlosmedios.htm $>\quad[30$ junio 2007]

(Mestres i Salud, 2007) Mestres i Salud, Laia. Los medios de comunicación y la educación. [En línea] Madrid: Educaweb, 1998. <http://www.educaweb.com/EducaNews/ interface/asp/web/NoticiesMostrar.asp?NoticialD=2206\& SecciolD=2479> [12 julio 2007]

Morduchowicz, R. (2001). Los medios de comunicación y la educación: un binomio posible. // Revista Iberoamericana de Educación, número 26. [En línea] OEI: Madrid, 2001. < http://www.rieoei.org/rie26a05.htm> [12 julio 2007]

Pérez Tapia, José Antonio (2006). Tareas de la educación en la cultura digital: Parte I. // Educere, marzo 2006, volumen 10, $n^{\circ} .32$. p. 17-26. [En línea] Universidad de Los Andes: La Liria, 1996. <http://www.scielo.org.ve/scielo .php?script=sci_arttext\&pid=S1316-4910200600010000 $3 \& \operatorname{lng}=e s \& n r m=i s o>$. [15 junio 2007]

Serrano Cobos, Jorge (2006) Web 2.0 en las bibliotecas: el concepto Library 2.0. // Thinkepi, mayo 2006. [En línea] EPI: 2005. <http://www.thinkepi.net/repositorio/web-20en-las-bibliotecas-el-concepto-library-20/> [2 julio 2007]

Unión Europea (2006) Recomendación del Parlamento Europeo y del Consejo sobre las competencias clave para el aprendizaje permanente. [En línea] DOCE, 3012-2006. <http://eur-lex.europa.eu/LexUriServ/site/es/oj/ 2006/l_394/I_39420061230es00100018.pdf> [6 julio 2007]. 